\title{
Quantum Effect on Elementary Process of Diffusion and Collective Motion of Brown Particles
}

\author{
Takahisa Okino \\ Applied Mathematics Department, Faculty of Science \& Engineering, Oita University, Oita, Japan \\ Email: okino@oita-u.ac.jp
}

How to cite this paper: Okino, T. (2018) Quantum Effect on Elementary Process of Diffusion and Collective Motion of Brown Particles. Journal of Modern Physics, 9, 1007-1028.

https://doi.org/10.4236/jmp.2018.95063

Received: March 12, 2018

Accepted: April 22, 2018

Published: April 25, 2018

Copyright $\odot 2018$ by author and Scientific Research Publishing Inc. This work is licensed under the Creative Commons Attribution International License (CC BY 4.0).

http://creativecommons.org/licenses/by/4.0/

\section{(c) (i) Open Access}

\begin{abstract}
The correlation between the Schrödinger equation and the diffusion equation revealed that the relation of material wave is not a hypothesis but an actual one valid in a material regardless of the photon energy. Using the relations of material wave and uncertain principle, the quantum effect on elementary process of diffusion is discussed. As a result, the diffusivity is obtained as a universal expression applicable to any problem of diffusion phenomena. The Gauss theorem in theory and the Kirkendall effect in experimentation reveal the necessity of the coordinate transformation for a diffusion equation. The mathematical method for solving an interdiffusion problem of many elements system is established. The phase shift of the obtained analytical solution indicates the correlation between the solutions of each diffusion equation expressed by a fixed coordinate system and by a moving coordinate system. Based on the coordinate transformation theory, some unsolved problems of diffusion theory are reasonably solved and also some new important findings are discussed in relation to matters in the existing diffusion theory.
\end{abstract}

\section{Keywords}

Diffusion, Quantum Effect, Brown Motion, Material Wave, Markov Process

\section{Introduction}

The diffusion equation is one of the most fundamental and important equations in physics. In history, Fick [1] applied the heat conduction equation proposed by Fourier [2] in 1822 to the diffusion phenomena in 1855 as it had been. In addition, the diffusion equation is also applicable to the so-called Brown [3] problems relevant to the Markov [4] process in mathematics. The mathematical me- 
thod expanded here is thus applicable to the Brown problems in various science fields such as functional materials science, information science, robotic science, life science, social science, and so on [5]-[10]. The diffusion problems themselves have been widely and actively investigated by analyzing the diffusion equation in accordance with the industrial requirements for the development of new useful materials [11]-[16].

The behavior of a micro particle should be essentially investigated by using the Schrödinger [17] equation. On the other hand, in relation to the collective motion of micro particles, the behavior of their concentration has been investigated by solving the diffusion equation. The concentration profile of micro particles in a material is governed by a diffusivity of the given diffusion equation, and the diffusivity depends on an interaction between a micro particle and the other particles near the micro particle itself. The behavior of diffusivity in detail should be thus investigated in the category of quantum mechanics.

In 1923, de Broglie [18] proposed a hypothesis, where he supposed that the equation valid in relation to the photon energy is also applicable to a material particle. The so-called relation of material wave gives a basis for applying quantum mechanics to material particles and thus it is an extremely meaningful relation in quantum mechanics. The experimental evidence for the wave characteristic of a material particle was revealed after that, while the theoretical validity of equation for a material particle had not been revealed. In that situation, the Schrödinger equation was reasonably derived from the diffusion equation [19]. In the derivation process, the elementary quantity of diffusivity and the relation of material wave unrelated to the photon energy were reasonably obtained.

Using the relations of material wave and the uncertain principle, we could reasonably understand the elementary process of diffusion from a new viewpoint. We incorporated a kinetic potential into the Boltzmann factor [20] relevant to the existence probability of a micro particle in a material, and applied it to the elementary quantity of diffusivity. As a result, the actual diffusivity different from the existing theory within the category of classical mechanics and thermodynamics was obtained as a universal expression applicable to any material, regardless of the thermodynamic state.

For the well-known diffusion equation with a diffusivity $D$ given by

$$
\frac{\partial C}{\partial t}=\frac{\partial}{\partial x}\left\{D \frac{\partial C}{\partial x}\right\}
$$

for a concentration $C(t, x)$ in the time and space $(t, x)$, the Gauss theorem indicates that the diffusion flux of

$$
J(t, x)=-D \frac{\partial C}{\partial x}+J(t)+J_{\text {eq }}
$$

is mathematically valid because of $\partial / \partial x\left\{J(t)+J_{\text {eq }}\right\}=0$ [21]. Here, it is considered that $J(t)$ corresponds to a movement of diffusion region space, which is composed of the so-called vacancies and interstices among micro particles in a 
material, and that $J_{\text {eq }}$ independent of $t$ and $x$ means an intrinsic diffusion flux in the thermal equilibrium state [22]. In other words, the existence of $J(t)$ relates to a volume variation of diffusion region, and the diffusion equation still holds as a material conservation law even if the volume of diffusion region changes during a diffusion treatment.

The definition of diffusivity relevant to a relative motion between micro particles indicates that the coordinate origin of diffusion equation should be usually set at a point in the diffusion region space. The existence of $J(t)$ indicates that the diffusion equation should be generally expressed by a moving coordinate system. On the other hand, the concentration profiles obtained in a laboratory are always observed by a fixed coordinate system in the diffusion region outside. The experimental result known as Kirkendall [23] effect also reveals that the coordinate transformation of diffusion equation is necessary for understanding the diffusion phenomena [24]. Nevertheless, the matter relevant to coordinate transformation of diffusion equation had never been discussed until recently in a long history of the diffusion theory. From a viewpoint of the coordinate transformation theory, the fundamental theory of diffusion is concretely and systematically discussed in the present work.

Solving the interdiffusion problems of many elements system is extremely important for the development of new useful materials. In general, the diffusivity depends on the concentration in the interdiffusion problems and the diffusion Equation (1) becomes then generally nonlinear differential equation. It had been thus considered that the mathematical solutions of Equation (1) are then impossible. However, recently the general solutions of Equation (1) were obtained as analytical expressions [25]. Their solutions are obtained at a high temperature during the diffusion treatment. On the other hand, the experimental results are observed at a room temperature after the diffusion treatment. When we compare analytical results with experimental results, therefore, we must take account of the influence of difference between these temperatures because of a movement of the diffusion region space during the temperature fall. That means a phase shift of the analytical solution, which is essentially relevant to the coordinate transformation. Incorporating such a shift effect into the obtained solution, the experimental results were reasonably reproduced [26]. At the same time, the Kirkendall effect was also reasonably understood as a shift between coordinate systems regardless of the concept of intrinsic diffusion [24] [26].

The coordinate transformation theory of a diffusion equation established that the diffusion equation of a moving coordinate system in the diffusion region inside is expressed as

$$
\frac{\partial C}{\partial t}=D \frac{\partial^{2} C}{\partial x^{2}}
$$

even if the diffusivity depends on the concentration. Further, it was concretely revealed that the solutions of Equation (3) agree with those of Equation (1) if we take account of the phase shift between their analytical solutions, and vice versa. 
Some new findings resulting from the coordinate transformation theory of diffusion equation and also resulting from the general solutions obtained as analytical expressions are concretely and systematically discussed. Thus, the new findings obtained here will be not only widely useful but also indispensable for analyzing the actual diffusion problems in future, just because of extremely fundamental ones.

\section{Elementary Process of Diffusion and Diffusivity}

Hereafter, an abbreviate differential notation for an arbitrary independent variable $\xi$ and the well-known bracket notation of Dirac [27] for an arbitrary vector are used as follows

$$
\partial_{\xi}=\frac{\partial}{\partial \xi},\langle\tilde{\nabla}|=\left(\partial_{x}, \partial_{y}, \partial_{z}\right),\langle r|=(x, y, z)
$$

If an operator $Q$ is Hermite one, $\langle Q|=\{|Q\rangle\}^{\dagger}$ is valid in the Hermite conjugate $\dagger$. Here, the notation $\langle\tilde{\nabla}|$ is thus defined as $\langle\tilde{\nabla}|=-\{|\nabla\rangle\}^{\dagger}$ because of $\partial_{\xi}=-\left\{\partial_{\xi}\right\}^{\dagger}$.

The function $C\left(t_{j},\left|r_{j}\right\rangle\right)$ is defined as a normalized concentration where a diffusion particle in the initial time and space $\left(t_{0},\left|r_{0}\right\rangle\right)$ exists in the time and space $\left(t_{j},\left|r_{j}\right\rangle\right)$ after $j$ times jumps. Since it is considered that the jump-probability from $\left(t_{j-1},\left|r_{j-1}\right\rangle\right)$ to $\left(t_{j},\left|r_{j-2}\right\rangle\right)$ is equivalent to that from $\left(t_{j-1},\left|r_{j-1}\right\rangle\right)$ to $\left(t_{j},\left|r_{j}\right\rangle\right)$ in the isotropic space, the relation of

$$
C(t+\Delta t,|r\rangle)=\{C(t,|r\rangle-|\Delta r\rangle)+C(t,|r\rangle+|\Delta r\rangle)\} / 2
$$

is then valid in the Markov process [4]. The Taylor expansion of both sides of Equation (4) yields

$$
\partial_{t} C=\frac{(\Delta r)^{2}}{2 \Delta t}\langle\tilde{\nabla} \mid \nabla C\rangle
$$

where $\left.\Delta r=|| r_{j}\right\rangle-\left|r_{j-1}\right\rangle|=\| \Delta r\rangle \mid[28]$.

From the averaged $\Delta t$ and $\Delta r$ for a micro particle with mass $m$ in a material, it is considered that $(\Delta r)^{2} / 2 \Delta t$ becomes a finite value of

$$
D=\frac{(\Delta r)^{2}}{2 \Delta t}=\frac{\Delta r p}{2 m}
$$

where $p$ is a momentum of the micro particle, and $D$ called the diffusivity may depend on the configuration of micro particles near the time and space $\left(t_{j},\left|r_{j}\right\rangle\right)$. The basic equation of diffusion is then expressed as

$$
\partial_{t} C=D\langle\tilde{\nabla} \mid \nabla C\rangle,
$$

where the coordinate origin of Equation (7) expressing the relative motion between diffusion particles should be generally set at a point of the diffusion region space.

The behavior of a micro particle should be generally controlled by the quantum theory. The correlation between the Schrödinger equation and the diffusion 
equation was thus investigated in the previous work as shown in Figure 1 [19]. As a result, the diffusivity of

$$
D_{0}=\hbar / 2 m
$$

for a micro particle with mass $m$ is then obtained using the Planck constant $\hbar(=h / 2 \pi)$ of the characteristic constant of a micro particle.

The collective motion of micro particles in a material results essentially from the statistical behavior of collision between a micro particle and the nearest particles surrounding the micro particle itself, where the statistical behavior is directly relevant to a jumping problem of a micro particle in a material. Since the diffusivity value of Equation (6) obtained from a jumping problem of a micro particle is equivalent to that of Equation (8) obtained from corresponding the diffusion equation to the Schrödinger equation in a collision problem, the relation of

$$
\Delta r p=\hbar
$$

is valid. When a micro particle exists as a wave packet of wave length $\lambda$ in the smallest local space $\Delta r$, the relation of

$$
\lambda=2 \pi \Delta r
$$

must be valid, while it exists as a particle if $0<\Delta r<\lambda / 2 \pi$. Here, the relation of

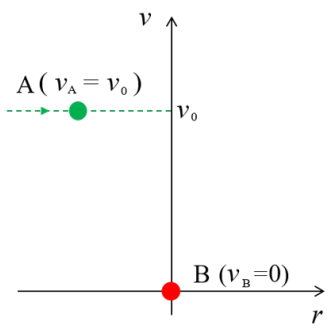

(a)

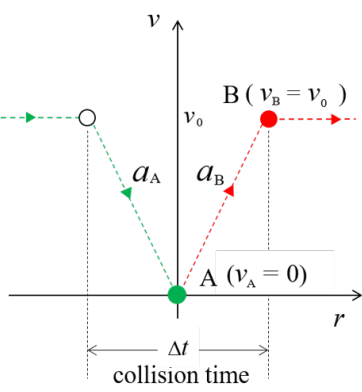

(b)

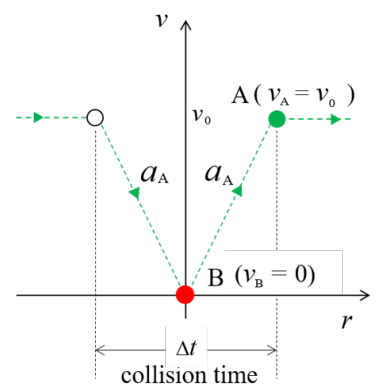

(c)

Figure 1. An elastic collision between micro particles of the same kind. (a) A state before the collision: The micro particle A moves with a velocity $v_{\mathrm{A}}=v_{0}$ and the particle $\mathrm{B}$ is in the rest state of the velocity $v_{\mathrm{B}}=0$; (b) A state before and after the collision: If we can identify the difference between micro particles $\mathrm{A}$ and $\mathrm{B}$, the particle A reaches $v_{\mathrm{A}}=0$ from a velocity $v_{\mathrm{A}}=v_{0}$ with the acceleration $a_{\mathrm{A}}=-\infty$ for $\Delta t \rightarrow 0$. The particle $\mathrm{B}$ reaches $v_{\mathrm{B}}=v_{0}$ from the velocity $v_{\mathrm{B}}=0$ with the acceleration $a_{\mathrm{B}}=\infty$ for $\Delta t \rightarrow 0$; (c) A state before and after the collision: If we cannot identify the difference between them, it seems that the particle A reaches $v_{\mathrm{A}}=0$ from the velocity $v_{\mathrm{A}}=v_{0}$ with the acceleration $a_{\mathrm{A}}=-\infty$ for $\Delta t \rightarrow 0$, and subsequently it reaches $v_{\mathrm{A}}=v_{0}$ again from the velocity $v_{\mathrm{A}}=0$ with the acceleration $a_{\mathrm{A}}=\infty$ for $\Delta t \rightarrow 0$. In the process of $v_{\mathrm{A}}=0 \rightarrow v_{\mathrm{A}}=v_{0}$, the definition of acceleration indicates that $t \rightarrow i t$ is valid because of $a=\Delta v / \Delta t=\Delta r /(\Delta t)^{2}$. In that case, it seems that the particle $\mathrm{B}$ is still in the rest state during the collision process. Letting the above situation (c) correspond to the elementary process of diffusion, the Schrödinger equation was reasonably derived from the diffusion equation (see Ref. [19]). 
material wave yielding

$$
p=h / \lambda .
$$

was reasonably obtained by using Equations (9) and (10) [19]. Fundamentally speaking, Equation (11) is a hypothesis proposed by de Broglie [18] in 1923, where he supposed that the equation relevant to the photon energy is also applicable to a material particle. Thus, it was revealed that the relation of material wave is reasonably valid in a material regardless of photon energy.

The relation of uncertain principle proposed by Heisenberg [29] given by

$$
\Delta r \Delta p \geq \hbar / 2
$$

is well known. The uncertain principle shows that a micro particle in a local space $\Delta r$ moves randomly with a momentum $\Delta p(\geq \hbar / 2 \Delta r)$ near the center of local space if $0<\Delta r<\lambda / 2 \pi$, because of the relation of material wave. It is thus considered that a Brown particle moves with a finite momentum $\Delta p$ in a local space $\Delta r$ and instantly jumps at a time from the local space to the nearest local space. In that situation, the above momentum $p$ of Equation (6) seems to be statistically equal to the momentum $\Delta p$. Thus, Equations (6) and (12) yield

$$
D \geq D_{\mathrm{E}} / 2 n(=\hbar / 4 m) \text { for } D_{\mathrm{E}}=5 \times 10^{2} N_{\mathrm{A}} \hbar=3.18 \times 10^{-8}\left[\mathrm{~m}^{2} \cdot \mathrm{s}^{-1}\right]
$$

where $N_{\mathrm{A}}$ and $n\left(=10^{3} \mathrm{mN}_{\mathrm{A}}\right)$ are the Avogadro constant and a molecular weight. Here, the value of $D_{\mathrm{E}}$ is an elementary quantity of diffusivity which is valid regardless of a kind of material and the thermodynamic state. Equation (13) shows that a micro particle in a material has an essential possibility for jumping from a local space to the nearest-neighbor one in accordance with the thermodynamic state.

In the existing theory of diffusion, it has been considered that the random movement of micro particles in a material is caused by a thermal fluctuation. In other words, it has been considered that the diffusion occurs when a vacancy is formed at the nearest-neighbor of a diffusion particle in a material through the thermal fluctuation. Here, when the radius $\Delta r$ of local space satisfies $\Delta r=\lambda / 2 \pi$ for a micro particle through the uncertain principle of Equation (12), the relation of material wave shows that the micro particle passes smoothly through an interstice between the nearest micro particles as not a particle image but as a wave image, even if the nearest vacancy does not exist [22]. In that situation, however, if a size $\Delta r$ of the nearest vacancy satisfies $\Delta r \geq \lambda / 2 \pi$, the concerned micro particle passes through the vacancy site still as a wave image and reaches a next coming local space, and it stays there as a particle if the size $\Delta r$ of the local space satisfies $\Delta r<\lambda / 2 \pi$ then. The diffusion phenomena occur through such an iteration mechanism in a material. Thus, the present theory shows that the vacancy near a diffusion particle is not necessary for the elementary process of diffusion. In other words, the basis of Brown motion in a material results from the relations of material wave and uncertain principle.

For the actual diffusivity, the dependences of a temperature $T$ and the coordi- 
nate system $(x, y, z)$ should be incorporated into $D_{0}=\hbar / 2 m$. The existence probability of a micro particle with a thermodynamic activation energy $Q$ in a material is well known as the Boltzmann factor $\exp \left[-Q / k_{\mathrm{B}} T\right]$, where $k_{\mathrm{B}}$ is the Boltzmann constant [20]. The internal force $f$ acts usually on a micro particle in a material, and further an external force $F$ may sometimes act on the diffusion field of an isolated system. Using the kinetic potential $U_{\mathrm{f}}$ and $U_{\mathrm{F}}$ of these forces and the energy $U_{0}$ relevant to the entropy of diffusion system, the potential energy $U=U_{\mathrm{f}}+U_{\mathrm{F}}+U_{0}$ is defined here also taking account of the case of $U_{\mathrm{f}}=0$ or $U_{\mathrm{F}}=0$. Thus, the new diffusivity expression [19] [22] applicable to an arbitrary material and thermodynamic state is obtained as

$$
D=D_{\mathrm{N}} \exp \left[(U-Q) / k_{\mathrm{B}} T\right] \text { for } D_{\mathrm{N}}=D_{\mathrm{E}} / n=5 \times 10^{2} N_{\mathrm{A}} \hbar / n .
$$

\section{Diffusion Equation and Diffusion Flux}

In the physical system for a material composed of $N(j=1,2, \cdots, N)$ elements in the region $V(x, y, z)$ within the closed surface $S(x, y, z)$, the Gauss theorem yields

$$
\iint_{S}\left\langle n \mid J^{j}(t, x, y, z)\right\rangle \mathrm{d} S=\iiint_{V}\left\langle\tilde{\nabla} \mid J^{j}(t, x, y, z)\right\rangle \mathrm{d} V
$$

for an arbitrary differentiable vector

$$
\left|J^{j}(t, x, y, z)\right\rangle=-D^{j}\left|\nabla C^{j}(t, x, y, z)\right\rangle .
$$

Here, $\langle n|$ is a unit vector perpendicular to the surface element $\mathrm{d} S$, and $C^{j}(t, x, y, z)$ and $D^{j}$ are the concentration and diffusivity for a material element $j$.

The Gauss theorem shows no more than a relation between the surface integral and volume integral in mathematics. In physics, however, the left-hand side of Equation (15) means the outflow of a material element $j$ per unit time from the closed surface $S(x, y, z)$, and thus it should be relevant to the increasing rate of $\partial_{t} C^{j}(t, x, y, z)$ as far as the material element $j$ is conserved within the closed surface $S(x, y, z)$. As a physical relation, therefore, using Equations (15) and (16) and the relation of

$$
\iint_{S}\left\langle n \mid J^{j}(t, x, y, z)\right\rangle \mathrm{d} S+\iiint_{V} \partial_{t} C^{j} \mathrm{~d} x \mathrm{~d} y \mathrm{~d} z=0
$$

the relation of material conservation law is obtained as

$$
\iint_{S}\left\{\int \partial_{t} C^{j} \mathrm{~d} x\right\} \mathrm{d} y \mathrm{~d} z=\iint_{S}\left\{\int\left\langle\tilde{\nabla} \| D^{j} \nabla C^{j}\right\rangle \mathrm{d} x\right\} \mathrm{d} y \mathrm{~d} z
$$

or

$$
\partial_{t} C=\langle\tilde{\nabla} \mid D \nabla C\rangle
$$

under the condition of no sink and source of the element $j$ within the closed surface $S(x, y, z)$.

The integral calculation of \{\} in Equation (17) shows that the variables $y$ and $z$ are accepted as constant values because of the characteristic of multiple 
integral calculations. Thus, Equation (17) yields the diffusion equation of

$$
\partial_{t} C^{j}(t, x)=\partial_{x}\left\{D^{j} \partial_{x} C^{j}(t, x)\right\} \text {. }
$$

The bracket \{\} in Equation (17) means an inflow flux from the surface element $\mathrm{d} y \mathrm{~d} z$. By defining the outflow flux as a plus value, the $x$ component $J_{x}^{j}$ of $\left\langle\tilde{J}^{j}\right|=\left(J_{x}^{j}, J_{y}^{j}, J_{z}^{j}\right)$ is then expressed as

$$
J_{x}^{j}=J^{j}(t, x)=-\int \partial_{t} C^{j}(t, x) \mathrm{d} x=-\int \partial_{x}\left\{D^{j} \partial_{x} C^{j}(t, x)\right\} \mathrm{d} x .
$$

In mathematics, Equation (20) yields the diffusion flux of

$$
J^{j}(t, x)=J_{\mathrm{F}}^{j}(t, x)+J^{j}(t)+J_{\text {eq }}^{j} \text { for } J_{\mathrm{F}}^{j}(t, x)=-D^{j} \partial_{x} C^{j}(t, x),
$$

where $J_{\mathrm{F}}^{j}(t, x)$ means the well-known Fick first law and $J^{j}(t)+J_{\text {eq }}^{j}$ is then a mathematical integral constant against $x$. In physics, $J^{j}(t)$ means a movement of a diffusion region space caused by the movement of diffusion particles. In a moving coordinate system setting the coordinate origin at a point in the diffusion region space, the flux should be physically accepted as $J^{j}(t)=0$. However, the case of $J^{j}(t) \neq 0$ must be considered for the fixed coordinate system of diffusion region outside. The flux $J_{\text {eq }}^{j}$ independent of $t$ and $x$ is an intrinsic one relevant to the Brown motion in the thermal equilibrium state. It plays an important role for understanding a self-diffusion mechanism [22].

Hereafter, we discuss such an interdiffusion problem in the time and space $(t, x)$ for a diffusion couple, since the generality of mathematical physics for the diffusion theory is still kept even if the simplest coordinate system of the time and space $(t, x)$ is used. We conceive a diffusion couple composed of a material $A$ and a material B with a uniform shape and the same cross section as shown in Figure 2. The material A is smoothly joined to the material $B$ at the initial interface $x=0$ then. Further, the materials A for $x<0$ and B for $x>0$ are composed of $L(\leq N)$ elements and $M(\leq N)$ elements, and there are $N$ elements in the diffusion region during a diffusion treatment.

The diffusion depth proportional to $\sqrt{D t}$ indicates that the concentration profile of diffusion at a low temperature agrees with that of diffusion at a high temperature, if the diffusion time is suitably long. This means that we can essentially analyze a diffusion problem under the condition of temperature where the variation of total particles on a cross section in the diffusion region is negligible during a thermal treatment. In the research field concerned, for an arbitrary $k$ between $1 \leq k \leq N$, it is widely accepted that the relations of

$$
\sum_{j=1}^{N} C^{j}(t, x)=1 \text { and } \sum_{j=1}^{N} \partial_{t} C^{j}(t, x)=0, \quad \partial_{x} C^{k}(t, x)=-\sum_{j=1, j \neq k}^{N} \partial_{x} C^{j}(t, x)
$$

is valid between the normalized concentrations of $N$ elements on a cross section in the diffusion region of $x_{\mathrm{A}} \leq x \leq x_{\mathrm{B}}$. Substituting Equation (22) into the diffusion Equation (19), the relation of

$$
\partial_{x} C^{k}(t, x)=-\sum_{j=1, j \neq k}^{N} \frac{D^{j}}{D^{k}} \partial_{x} C^{j}(t, x)
$$




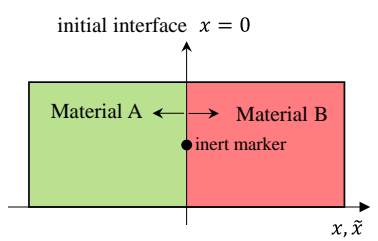

(a)

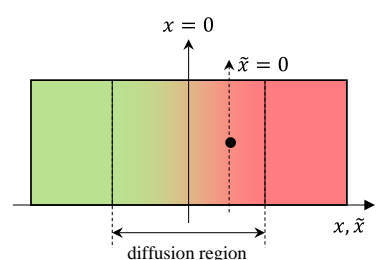

(b)

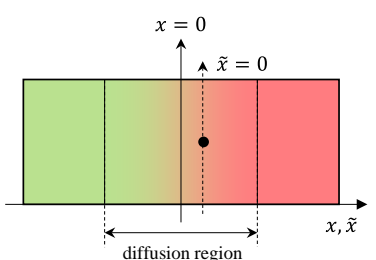

(c)

Figure 2. Schematic figure of interdiffusion phenomena. The material A is smoothly joined to the material $\mathrm{B}$ at the initial interface $x=0$. The materials $\mathrm{A}$ for $x<0$ and $\mathrm{B}$ for $x>0$ are composed of $L(\leq N)$ elements and $M(\leq N)$ elements, and there are $N$ elements in the diffusion region during a diffusion treatment. The coordinate systems of $(t, x)$ and $(\tilde{t}, \tilde{x})$ are set at a point of the mass center of diffusion particles and at a point of space on the initial interface between materials $A$ and $B$, respectively. The relation between their coordinate systems resulting from the movement of diffusion region space is conceived as $t=\tilde{t}$ and $x=\tilde{x}+\int_{0}^{t} v \mathrm{~d} \tilde{t}$ using a velocity $v$ of the origin of $(\tilde{t}, \tilde{x})$ against the origin of $(t, x)$. The notation $\bullet$ means an inert marker set at the coordinate origin of $(\tilde{t}, \tilde{x})$. The partial figures (a), (b) and (c) represent the initial state at a room temperature, a state at a high temperature during diffusion treatment and a state at a room temperature after diffusion treatment, respectively. See Ref. [26] for further details.

is valid in the differential Equation (19).

Since Equations (22) and (23) are simultaneously valid as identical equations, the relation of

$$
\tilde{D}=D^{1}=D^{2}=\cdots=D^{N}
$$

must be valid in the differential Equation (19) then [24]. Using the so-called interdiffusion coefficient $\tilde{D}$, Equation (19) is then rewritten as

$$
\partial_{t} C^{j}(t, x)=\partial_{x}\left\{\tilde{D} \partial_{x} C^{j}(t, x)\right\}=\left\{\tilde{D} \partial_{x}+\left(\partial_{x} \tilde{D}\right)\right\} \partial_{x} C^{j}(t, x) \text { for } j=I, I I, \cdots, N \text {. }
$$

Here, the interdiffusion coefficient $\tilde{D}$ has been accepted as an actual one in the existing theory of diffusion. However, $\tilde{D}$ of Equation (24) is not a real quantity but a mathematical operator valid only in the differential Equation (25) and acts in common on each element in the diffusion field under the condition of Equation (22).

For the development of new useful materials, solving the interdiffusion problems of many elements system is extremely important. For the interdiffusion problem of many elements system, the present analytical method resulted in solving Equation (25) in accordance with the initial and/or boundary conditions of each element under the bound condition of Equation (22) [24]. However, it had been considered for a long time that the mathematical solutions of Equation (19) are impossible.

Using the initial and/or boundary values $D_{\mathrm{A}}^{j}, C_{\mathrm{A}}^{j}$ at $x=x_{\mathrm{A}}$ and $D_{\mathrm{B}}^{j}, C_{\mathrm{B}}^{j}$ at $x=x_{\mathrm{B}}$ in the diffusion region $x_{\mathrm{A}} \leq x \leq x_{\mathrm{B}}$ for $\tilde{D}$ and $C^{j}(t, x)$ in the diffusion Equation (25), the general solutions were for the first time mathematically obtained as (see Ref. [25]) 


$$
\begin{gathered}
D^{j}(t, x)=\frac{D_{\mathrm{A}}^{j}+D_{\mathrm{B}}^{j}}{2}-\frac{D_{\mathrm{A}}^{j}-D_{\mathrm{B}}^{j}}{2} \operatorname{erf}\left(\frac{x}{2 \sqrt{D_{\mathrm{int}}^{j} t}}+\alpha^{j}\right), \\
C^{j}(t, x)=\frac{C_{\mathrm{A}}^{j}+C_{\mathrm{B}}^{j}}{2}-\frac{C_{\mathrm{A}}^{j}-C_{\mathrm{B}}^{j}}{2} \operatorname{erf}\left(\frac{x}{2 \sqrt{D_{\mathrm{int}}^{j} t}}+\beta^{j}\right),
\end{gathered}
$$

where $D_{\mathrm{int}}^{j}=D_{\mathrm{int+}}^{j}=\left(D_{\mathrm{A}}^{j}+D_{\mathrm{B}}^{j}\right) / 2$ for $x \geq 0, D_{\mathrm{int}}^{j}=D_{\mathrm{int}-}^{j}=\sqrt{D_{\mathrm{A}}^{j} D_{\mathrm{B}}^{j}}$ for $x<0$ and

$$
\begin{gathered}
\alpha^{j}=\operatorname{erf}^{-1}\left(\frac{D_{\mathrm{A}}^{j}+D_{\mathrm{B}}^{j}}{D_{\mathrm{A}}^{j}-D_{\mathrm{B}}^{j}}-\frac{2}{\ln D_{\mathrm{A}}^{j}-\ln D_{\mathrm{B}}^{j}}\right), \\
\beta^{j}=\alpha^{j}-\left(\sqrt{D_{\mathrm{A}}^{j}}-\sqrt{D_{\mathrm{B}}^{j}}\right) /\left(\sqrt{D_{\mathrm{A}}^{j}}+\sqrt{D_{\mathrm{B}}^{j}}\right) .
\end{gathered}
$$

We confirmed that the solutions of Equations (26) and (27) agree well with results of the empirical Boltzmann Matano method [30] [31]. Further, the validity of their solutions was also actually confirmed by applying them to the experimental results for the binary system interdiffusion [23] [26].

In case of a binary system interdiffusion, for example, the relations between diffusivities and between diffusion fluxes yielding

$$
\tilde{D}=D^{\mathrm{I}}=D^{\mathrm{II}}, \quad \tilde{J}^{\mathrm{I}}+\tilde{J}^{\mathrm{II}}=-\tilde{D} \partial_{x}\left\{C^{\mathrm{I}}+C^{\mathrm{II}}\right\}=0
$$

had been misunderstood as actual ones in the existing diffusion theory. However, they should be valid only in the differential Equation (25), since the initial values have not yet been taken into account. On the other hand, the relations of

$$
D^{\mathrm{I}} \neq D^{\mathrm{II}}, \quad J^{\mathrm{I}}+J^{\mathrm{II}}=-\left\{D^{\mathrm{I}} \partial_{x} C^{\mathrm{I}}+D^{\mathrm{II}} \partial_{x} C^{\mathrm{II}}\right\} \neq 0
$$

taking account of initial values are naturally valid in case of using the solutions of Equations (26) and (27). It is obvious that the Kirkendall effect is caused by Equation (29) [23] [32]. Nevertheless, Equation (28) had been mistaken as a relation between actual diffusion fluxes in the history of diffusion. As a result, the intrinsic diffusion coefficients $D_{\mathrm{INT}}^{\mathrm{I}}$ and $D_{\mathrm{INT}}^{\mathrm{II}}$ satisfying $D_{\mathrm{INT}}^{\mathrm{I}} \neq D_{\mathrm{INT}}^{\mathrm{II}}$ were newly conceived without reasonable evidence in order to understand the Kirkendall effect in those days.

As discussed in Section 2, the diffusivity correlates with a jumping frequency of one micro particle in a material. There is only one diffusion particle at a point of time and space $(t, x, y, z)$ and the diffusion particle has then statistically a value of jumping frequency. Thus, one element has only one diffusivity value in the diffusion field concerned. In the present diffusion field, therefore, the diffusivities of elements I and II are just $D^{\mathrm{I}}$ and $D^{\mathrm{II}}$ and the other diffusivities relevant to such diffusion mechanisms as an interdiffusion and an intrinsic diffusion are actually nonexistent from the beginning.

\section{Coordinate Transformation Theory of Diffusion Equation}

The existence of $J^{j}(t)$ in Equation (21) indicates that we must take account of the difference between the diffusion equation of coordinate system set a point in 
the diffusion region space expressing the relative motion between micro particles and that of coordinate system set a point in the laboratory system outside the diffusion region. Based on the mathematical and/or physical consideration, the problems of coordinate system of diffusion equation are discussed in the following.

In relation to the interdiffusion problem, we conceived a raft model [32] where the relative motion of two parsons $\mathrm{A}$ with a mass $m_{\mathrm{A}}$ and $\mathrm{B}$ with a mass $m_{\mathrm{B}}$ on the raft with a mass $M_{\mathrm{R}}$ floating on the still water was discussed as an isolated system. In that case, the mass center of $M_{\mathrm{R}}, m_{\mathrm{A}}$ and $m_{\mathrm{B}}$ is immovable against a fixed point outside the isolated system even if each parson moves freely on the raft. In other words, the raft moves with a velocity against a fixed point outside the raft like the mass center is immovable against a fixed point outside the raft. The coordinate origin of $(\tilde{t}, \tilde{x})$ is set at a point of the mass center of raft itself, and the coordinate origin of $(t, x)$ is set at a point of the mass center of the parsons $\mathrm{A}$ and $\mathrm{B}$, and the coordinate origin $(\tau, \xi)$ is set at a point outside the raft. We conceive that the relation of $x=\tilde{x}=\xi=0$ is valid in the initial sate at $t=\tilde{t}=\tau=0$ then.

As can be easily seen, we can let the raft model correspond to interdiffusion phenomena in the diffusion couple shown in Figure 2. In that case, the raft and the parsons correspond to the diffusion region space and diffusion particles, respectively. The coordinate origin of $(\tilde{t}, \tilde{x})$ is set at a point of diffusion region space on the initial interface, and we may then conceive that the coordinate origin of $(\tilde{t}, \tilde{x})$ is set a point of a virtual inert marker on the initial interface at $\tilde{X}=0$, since the inert marker does not interact with the diffusion field because of its inert characteristic. The coordinate origin of $(t, x)$ is set at a point of the mass center of diffusion particles on the initial interface at $x=0$. The coordinate origin of $(\tau, \xi)$ is set at a point of diffusion region outside. Here, in relation to what the diffusion region space has no mass, the coordinate origin of $(t, x)$ is immovable against the coordinate origin of $(\tau, \xi)$, if any external force does not act on the diffusion particles in the isolated system. In other words, the coordinate system $(t, x)$ is equivalent to the coordinate system $(\tau, \xi)$ then.

When the interaction $f$ in the diffusion field of an isolated system influences on diffusion particles under the condition of an external force $F=0$, a velocity $v_{i}(t)$ of the coordinate origin $\tilde{x}=0$ against the coordinate origin $x=0$ is defined as

$$
\tilde{X}=x+x_{\text {stt }} \text { for } \tilde{t}=t \text { and } x_{\text {stt }}=-\int_{0}^{\tilde{t}} v_{i} \mathrm{~d} t .
$$

Here, the coordinate origin $(t, x)$ set at a point of mass center of diffusion particles on the initial interface is then immovable because of $F=0$. As a matter of convenience, the suffix $j$ expressing an element is removed because of no meaning in the essential theory in the following. Equation (19) is then transformed into

$$
\partial_{\tilde{t}} C(\tilde{t}, \tilde{x})=\partial_{\tilde{x}}\left\{D \partial_{\tilde{x}} C(\tilde{t}, \tilde{x})-v_{i} C(\tilde{t}, \tilde{x})\right\} .
$$


Here, the term $v_{i} C(\tilde{t}, \tilde{x})$ means that the concentration-distance curve moves in parallel to the $x$ axis. The Gauss theorem shows that the diffusion flux of

$$
J(\tilde{t}, \tilde{x})=-D \partial_{\tilde{x}} C(\tilde{t}, \tilde{x})+v_{i} C(\tilde{t}, \tilde{x})+J_{\text {eq }}
$$

is valid then. Further, the Gauss theorem shows that the diffusion flux of the coordinate $(t, x)$ is expressed as

$$
J(t, x)=-D \partial_{x} C(t, x)+J(t, x)+J_{\text {eq }},
$$

since the coordinate origin $x=0$ is immovable.

Substituting Equation (14) into Equation (30) yields

$$
\begin{aligned}
\partial_{\tilde{t}} C(\tilde{t}, \tilde{x}) & =D \partial_{\tilde{x}}^{2} C(\tilde{t}, \tilde{x})+\left\{\left(\partial_{\tilde{x}} D\right)-v_{i}\right\} \partial_{\tilde{x}} C(\tilde{t}, \tilde{x}) \\
& =D \partial_{\tilde{x}}^{2} C(\tilde{t}, \tilde{x})-\left(D f / k_{\mathrm{B}} T+v_{i}\right) \partial_{\tilde{x}} C(\tilde{t}, \tilde{x})
\end{aligned}
$$

because of $f=-\partial_{\tilde{x}} U$ and $F=0$. Equation (6) shows that the diffusivity gradient $\partial_{\tilde{\chi}} D$ is a velocity itself of a diffusion particle. This means that the relation of

$$
\left(D f / k_{\mathrm{B}} T+v_{i}\right) \partial_{\tilde{x}} C(\tilde{t}, \tilde{x})=\partial_{\tilde{x}}\left(D f / k_{\mathrm{B}} T+v_{i}\right) C(\tilde{t}, \tilde{x})
$$

is valid. A diffusion particle and a local space in the diffusion field change places with each other in the one-to-one correspondence. Thus, the diffusion region space moves with the quite reverse velocity of what each diffusion particle moves with a velocity at each point $(t, x)$ on the concentration-distance curve. As a result, the relation of

$$
D f / k_{\mathrm{B}} T+v_{i}=0
$$

should be valid in the diffusion field. The diffusion particles then randomly move in accordance with the relation of

$$
\partial_{\tilde{t}} C(\tilde{t}, \tilde{x})=D \partial_{\tilde{x}}^{2} C(\tilde{t}, \tilde{x}),
$$

satisfying the parabolic law. The relations between solutions of Equations (19) and (35) given by

$$
C(\tilde{t}, \tilde{x})=C\left(t, x+x_{\mathrm{sft}}\right), \quad D(\tilde{t}, \tilde{x})=D\left(t, x+x_{\mathrm{sft}}\right) \text { and } J(\tilde{t}, \tilde{x})=J\left(t, x+x_{\mathrm{sft}}\right)
$$

are valid then.

Further, as an especial case, if we accept the same relation of $f=-k v_{i}$ that Langevin [33] as well as Einstein [28] used for investigating the Brown motion, Equation (34) gives the same result as

$$
D=k_{\mathrm{B}} T / k
$$

called the Einstein equation. Equation (37) gives evidence for the validity of the present diffusion theory, as far as equation (37) is valid in a material.

When an external force $F$ influences on the diffusion region from the diffusion region outside under the condition of $f=0$, a velocity $v_{e}(\tau)$ of the coordinate origin $x=0$ against the coordinate origin $\xi=0$ is defined as $x=\xi+\xi_{\text {st }}$ for $t=\tau$ and $\xi_{\text {stt }}=-\int_{0}^{t} v_{e} \mathrm{~d} \tau$, because of the movement of initial mass center through the external force. The diffusion equation of a fixed coor- 
dinate system in the diffusion region outside yielding

$$
\partial_{\tau} C(\tau, \xi)=\partial_{\xi}\left\{D \partial_{\xi} C(\tau, \xi)\right\}
$$

is transformed into the moving coordinate system $(t, x)$ given by

$$
\partial_{t} C(t, x)=\partial_{x}\left\{D \partial_{x}-v_{e}\right\} C(t, x)=D \partial_{x}^{2}(t, x)-\left(D F / k_{\mathrm{B}} T+v_{e}\right) \partial_{x} C(t, x),
$$

because of $F=-\partial_{\tilde{x}} U$ and $f=0$. In accordance with the same mechanism as the above position exchange between a diffusion particle and a local space, Equation (39) is also rewritten as

$$
\partial_{t} C(t, x)=D \partial_{x}^{2} C(t, x) .
$$

Equation (39) shows that the diffusion flux of

$$
J(t, x)=-D \partial_{x} C(t, x)+v_{e} C(t, x)+J_{\text {eq }}
$$

is valid then. Equation (41) corresponds to the diffusion flux in the laboratory system yielding

$$
J(\tau, \xi)=-D \partial_{\xi} C(\tau, \xi)+J(\tau)+J_{\text {eq }} .
$$

When a large external force $F$ satisfying the relation of

$$
F \gg k_{\mathrm{B}} T\left|\partial_{x}^{2}(t, x) / \partial_{x} C(t, x)\right| / D
$$

exists in the diffusion field, substituting Equation (14) into Equation (38) shows that the physical system concerned is not a diffusion problem from the start.

Based on the above analysis, it is found that there are 4 cases for relations between solutions of Equations (19), (35) and (38) as follows:

$$
\begin{cases}(\text { a }) C(\tilde{t}, \tilde{x})=C(t, x), \partial_{\tilde{x}} D(\tilde{t}, \tilde{x})=\partial_{x} D(t, x)=0 & \text { for } f=F=0 \\ \text { (b) } C(\tilde{t}, \tilde{x})=C\left(t, x+x_{\text {sft }}\right), D(\tilde{t}, \tilde{x})=D\left(t, x+x_{\text {stt }}\right) & \text { for } f \neq 0, F=0 \\ \text { (c) } C(t, x)=C\left(\tau, \xi+\xi_{\text {sft }}\right), D(t, x)=D\left(\tau, \xi+\xi_{\text {sft }}\right) & \text { for } f=0, F \neq 0 \\ \text { (d) } C(\tilde{t}, \tilde{x})=C\left(\tau, \xi+x_{\text {sft }}+\xi_{\text {sft }}\right), D(\tilde{t}, \tilde{x})=D\left(\tau, \xi+x_{\text {sft }}+\xi_{\text {sft }}\right) & \text { for } f \neq 0, F \neq 0\end{cases}
$$

The basis of diffusion problems results from the relative motion between diffusion particles in the diffusion region space. Equation (43) reveals that the basic equation of diffusion should be expressed by a moving coordinate system. Here, the summarization in this section is shown in the following.

Hereinbefore, the coordinate transformation theory of diffusion equation was discussed using the coordinate systems $(\tilde{t}, \tilde{x}),(t, x)$ and $(\tau, \xi)$. In accordance with the usual notation, however, if we redefine the coordinate system $(t, x, y, z)$ as a moving one in the diffusion region inside, Equation (43) shows that the basic equation of diffusion should be expressed as

$$
\partial_{t} C=D\langle\tilde{\nabla} \mid \nabla C\rangle
$$

regardless of whether $D$ depends on a concentration or not, and of whether an internal or an external force influences on the diffusion field or not. As discussed in relation to the derivation of Equations (35) or (40), Equation (7) shows the so-called Brown motion of the isotropic jumping iteration then. 
On the other hand, if we also redefine the coordinate system $(t, x, y, z)$ as a fixed one, the diffusion equation is expressed as

$$
\partial_{t} C=\langle\tilde{\nabla} \mid D \nabla C\rangle \text {. }
$$

In case of the one dimensional space of Equations (7) and (18), the relations between solutions of Equation (7) $C_{m}(t, x), D_{m}(t, x)$ and those of Equation (18) $C_{f}(t, x), D_{f}(t, x)$, and further between their diffusion fluxes, $J_{m}(t, x)$ and $J_{f}(t, x)$ are then obtained as

$$
\begin{aligned}
& C_{m}(t, x)=C_{f}\left(t, x+\sigma_{\text {sft }}\right), \quad D_{m}(t, x)=D_{f}\left(t, x+\sigma_{\text {sft }}\right) \text { and } \\
& J_{m}(t, x)=J_{f}\left(t, x+\sigma_{\mathrm{sft}}\right) \text {, }
\end{aligned}
$$

where $\sigma_{\text {sft }}$ has a value of $\sigma_{\mathrm{sft}}=0, \sigma_{\mathrm{sft}}=x_{\mathrm{sft}}, \sigma_{\mathrm{sft}}=\xi_{\mathrm{sft}}, \sigma_{\mathrm{sft}}=x_{\mathrm{sft}}+\xi_{\mathrm{sft}}$ in accordance with Equation (43).

\section{Kirkendall Effect}

Under the condition of $F \ll k_{\mathrm{B}} T\left|\partial_{x}^{2}(t, x) / \partial_{x} C(t, x)\right| / D$, the behavior of diffusion particles shows the isotropic random movement. In that case, the shift effect $x_{\mathrm{sft}}$ of Equation (43) should depend only on the initial values of diffusivity and concentration in the isolated diffusion region. In the previous work [24] for interdiffusion problems of a $N$ elements system, the relation of

$$
x_{\text {sft }}=\frac{2}{\mu} \sum_{j=I}^{N} \sqrt{D_{\omega}^{j}}\left(C_{\mathrm{A}}^{j}-C_{\mathrm{B}}^{j}\right) \sqrt{t}
$$

satisfying the parabolic law was obtained, where $\omega \rightarrow \mathrm{A}$ if $C_{\mathrm{A}}^{j}>C_{\mathrm{B}}^{j}$ and $\omega \rightarrow \mathrm{B}$ if $C_{\mathrm{A}}^{j}<C_{\mathrm{B}}^{j}$ and the relation of diffusion length $\mu \sqrt{D t}$ is used here. The velocity $v_{i}$ between the coordinate origins of a moving coordinate system and that of a fixed one becomes

$$
v_{i}=\frac{1}{\mu} \sum_{j=I}^{N} \sqrt{D_{\omega}^{j}}\left(C_{\mathrm{A}}^{j}-C_{\mathrm{B}}^{j}\right) t^{-0.5} .
$$

In general, the diffusion experiment is performed at a high temperature of $T=T_{\mathrm{H}}$ during a time interval of $0 \leq t \leq t_{\mathrm{F}}$. The solutions of Equations (26) and (27) are ones at the temperature of $T=T_{\mathrm{H}}$ and the time $t=t_{\mathrm{F}}$ then. On the other hand, the diffusion region space in experimental results is generally in the thermal equilibrium state at a room temperature of $T=T_{\mathrm{R}}$ after the diffusion treatment. When we compare the solutions of diffusion equation with the experimental results, therefore, we must take account of a behavior of the diffusion region space caused by the temperature fall from $T=T_{\mathrm{H}}$ to $T=T_{\mathrm{R}}$, since the concentration profile is then shifted by its behavior.

The coordinate $(t, x)$ shown in Figure 2 is a fixed coordinate system equivalent to (b) in Equation (43). In that case, just after the diffusion treatment, vacancies in the diffusion region for $x>0$ become in the supersaturated state, while those in the diffusion region for $x<0$ become in the unsaturated state, and vice versa. It is thus considered that the supersaturated vacancies move to 
the diffusion region in the unsaturated state of vacancies so as to reach a thermal equilibrium state after the diffusion treatment. The diffusion region space during diffusion treatment moves at the steady temperature of $T=T_{\mathrm{H}}$, while it moves during the temperature fall from $T=T_{\mathrm{H}}$ to $T=T_{\mathrm{R}}$ after the diffusion treatment. It may be then considered that the diffusion region space during the temperature fall interacts with the nearest space of the specimen surface.

As shown in Figure 2, the position of coordinate origin $\tilde{X}=0$ denoted by $x=-x_{\text {sft }}$ on the $x$ axis at $t=t_{\mathrm{F}}$ returns not to the initial position $x=0$ but to $x=\Delta x_{\text {eff }}$. That is due to the reason why a part of the supersaturated vacancies move toward the specimen surface considered as a sink and/or source and they do not then move along the $x$ axis because of the movement perpendicular to the $x$ axis. It may be also considered that the vacancies generated at the specimen surface diffuse toward the diffusion region of the unsaturated vacancies. Further, there is also a question of whether a mass of inert marker is negligible or not, even if the inert characteristic is perfect. However, all these matters relevant to the diffusion region space do not give the essential influence on the concentration profile expressing the relative motion between diffusion particles, in other words, they appear as a phase shift of the analytical solutions obtained in the moving coordinate system [26]. This gives just evidence for the necessity of coordinate transformation theory. Although the behavior of diffusion region space is considerably complicated in that situation, the relation of $x=\Delta x_{\text {eff }}$ is just the so-called Kirkendall effect itself.

The validity of theory mentioned here was concretely confirmed in comparison with the experimental results of diffusion couple with an inert marker [23] [26]. In addition, as far as the velocity of Equation (46) is not zero, the shift $x_{\text {stt }}$ appears in the obtained solutions at the temperature of $T=T_{\mathrm{H}}$ and $t=t_{\mathrm{F}}$, even if the Kirkendall effect $\Delta x_{\text {eff }}$ is approximately zero at the temperature of $T=T_{\mathrm{R}}$ in accordance with a material characteristic during the temperature fall.

Although the theoretical equation of Kirkendall effect $\Delta x_{\text {eff }}$ is given by

$$
\Delta x_{\text {eff }}=\frac{2}{\mu} \sum_{j=I}^{N} \sqrt{D_{\omega}^{j}}\left(C_{\mathrm{A}}^{j}-C_{\mathrm{B}}^{j}\right) \sqrt{t_{\mathrm{eff}}},
$$

we cannot understand a reasonable method at present for determining $t_{\text {eff }}$ from the given diffusion problems.

\section{Solutions in the Parabolic Space}

Equation (38) is applicable to the coordinate system $(\tau, \xi)$ in diffusion region outside. In that case, Boltzmann revealed that the equation of

$$
-\frac{\zeta}{2} \frac{\mathrm{d} C}{\mathrm{~d} \zeta}=\frac{\mathrm{d}}{\mathrm{d} \zeta}\left(D \frac{\mathrm{d} C}{\mathrm{~d} \zeta}\right)
$$

is valid in the parabolic space under the condition of parabolic law $\zeta=\xi / \sqrt{\tau}$ [30]. Equation (47) had not been mathematically solved for a long time. In that situation, recently Equation (47) was transformed into the relation of diffusion 
flux in the parabolic space given by [25] [34]

$$
J(\zeta)=-D(\zeta) \frac{\mathrm{d} C(\zeta)}{\mathrm{d} \zeta},
$$

where

$$
J(\zeta)=-J_{0} \exp \left[-\int_{0}^{\zeta} \frac{\eta}{2 D(\eta)} \mathrm{d} \eta\right] \text { for } J_{0}=\left.D(\zeta) \frac{\mathrm{d} C(\zeta)}{\mathrm{d} \zeta}\right|_{\zeta=0} .
$$

On the other hand, the dependence of diffusivity on the concentration means

$$
\frac{\mathrm{d} C}{\mathrm{~d} \zeta}=\frac{\partial C}{\partial \zeta}+\frac{\partial C}{\partial D} \frac{\partial D}{\partial \zeta}
$$

in mathematics. By solving simultaneously Equations (48) and (49), as a result, the solutions of Equations (26) and (27) were mathematically obtained [25] [34].

Here, it was revealed in Section 4 that Equation (38) corresponds to Equation (40) expressed by a moving coordinate system $(t, x)$ in the diffusion region inside, even if the diffusivity depends on the concentration. In that case, Equation (40) is transformed into

$$
-\frac{\lambda}{2} \frac{\mathrm{d} C}{\mathrm{~d} \lambda}=D \frac{\mathrm{d}^{2} C}{\mathrm{~d} \lambda^{2}} \text { for } \lambda=x / \sqrt{t} .
$$

Integrating directly Equation (50) with respect to $\lambda$ and using a integral constant $A_{2}$, the relation of

$$
\frac{\mathrm{d} C}{\mathrm{~d} \lambda}=A_{2} \exp \left[-\int \frac{\lambda}{2 D} \mathrm{~d} \lambda\right]
$$

is obtained. Further, integrating Equation (51) yields

$$
C=A_{1}+A_{2} \int \exp \left[-\int \frac{\lambda}{2 D} \mathrm{~d} \lambda\right] \mathrm{d} \lambda,
$$

where $A_{1}$ is an integral constant. Using a diffusivity $D_{\text {int }}$ independent of $\lambda$ and a correction parameter $\varepsilon_{1}$ for Equation (52), the approximate relation of

$$
\int \frac{\lambda}{2 D} \mathrm{~d} \lambda=\frac{\left(\lambda+\varepsilon_{1}\right)^{2}}{4 D_{\mathrm{int}}}
$$

is derived (see Ref. [25]). Further, using the same initial values of concentration as Equation (27), the solution of

$$
C(\lambda)=\frac{C_{\mathrm{A}}+C_{\mathrm{B}}}{2}-\frac{C_{\mathrm{A}}-C_{\mathrm{B}}}{2} \operatorname{erf}\left\{\frac{\lambda}{2 \sqrt{D_{\mathrm{int}}}}+\varepsilon\right\} \text { for } \varepsilon_{1}=2 \sqrt{D_{\mathrm{int}}} \varepsilon
$$

is obtained.

The solution of diffusivity of

$$
D(\lambda)=\frac{D_{\mathrm{A}}+D_{\mathrm{B}}}{2}-\frac{D_{\mathrm{A}}-D_{\mathrm{B}}}{2} \operatorname{erf}\left(\frac{\lambda}{2 \sqrt{D_{\mathrm{int}}}}+\varepsilon+\left(\sqrt{D_{\mathrm{A}}}-\sqrt{D_{\mathrm{B}}}\right) /\left(\sqrt{D_{\mathrm{A}}}+\sqrt{D_{\mathrm{B}}}\right)\right)(54)
$$

is also obtained by substituting Equation (53) into the equation expressing the dependence of diffusivity on concentration [26] given by 


$$
D=\frac{D_{\mathrm{A}}+D_{\mathrm{B}}}{2}-\frac{D_{\mathrm{A}}-D_{\mathrm{B}}}{2} \operatorname{erf}(f(C))
$$

where

$$
f(C)=\operatorname{erf}^{-1}\left\{\frac{C_{\mathrm{A}}+C_{\mathrm{B}}}{C_{\mathrm{A}}-C_{\mathrm{B}}}-\frac{2 C(\lambda)}{C_{\mathrm{A}}-C_{\mathrm{B}}}\right\}+\left(\sqrt{D_{\mathrm{A}}}-\sqrt{D_{\mathrm{B}}}\right) /\left(\sqrt{D_{\mathrm{A}}}+\sqrt{D_{\mathrm{B}}}\right) .
$$

When we solved Equation (48) [25], we did not notice the necessity of coordinate transformation between the fixed coordinate system and the moving one for the diffusion theory in those days. The solutions expressed by Equations (26) and (27) are thus equivalent to the fixed coordinate system. Using the velocity $V$ of the coordinate origin of $(t, x)$ against the coordinate origin of $(\tau, \xi)$, the shift effect $\varepsilon_{\text {sft }}$ is given by

$$
\varepsilon_{\text {sft }}=\sigma_{\text {sft }} / 2 \sqrt{D_{\text {int }} t} \text { for } \sigma_{\text {sft }}=\int_{0}^{t} v \mathrm{~d} \tau
$$

in the parabolic space. In accordance with the previous investigation, therefore, the $\varepsilon$ value should be accepted as

$$
\varepsilon=\operatorname{erf}^{-1}\left(\frac{D_{\mathrm{A}}+D_{\mathrm{B}}}{D_{\mathrm{A}}-D_{\mathrm{B}}}-\frac{2}{\ln D_{\mathrm{A}}-\ln D_{\mathrm{B}}}\right)-\varepsilon_{\mathrm{sft}}-\left(\sqrt{D_{\mathrm{A}}}-\sqrt{D_{\mathrm{B}}}\right) /\left(\sqrt{D_{\mathrm{A}}}+\sqrt{D_{\mathrm{B}}}\right) .
$$

In other words, the following relations

$$
C\left(\lambda-\varepsilon_{\mathrm{stt}}\right)=C(\zeta) \text { and } D\left(\lambda-\varepsilon_{\mathrm{stt}}\right)=D(\zeta) .
$$

are valid in the present case.

By analyzing the diffusion Equations (48) and (50) in the parabolic space corresponding to Equations (38) and (40), it was revealed that Equation (44) is reasonably valid. Thus, it was found that analyzing the diffusion problem is satisfied by solving either Equation (7) or (18). In other words, the validity of coordinate transformation theory discussed here was revealed.

\section{Mathematical Misconceptions in the History of Diffusion}

In the diffusion field, the diffusivities relevant to such diffusion mechanisms as the self-diffusion, the impurity diffusion, the one-way diffusion, the interdiffusion, the intrinsic diffusion, and so on had been investigated in accordance with the given diffusion problems. In relation to the diffusivities mentioned here, some misconceptions in the existing diffusion theory are revealed in the following.

Based on the mathematical theory of differential equation, it was revealed in relation to Equation (24) that the diffusivity called the interdiffusion coefficient is not an actual one but a mathematical operator valid only in the differential equation, which acts in common on each of diffusion elements in the diffusion system. At the same time, it was revealed that there is no reasonable necessity for introducing the concept of intrinsic diffusion into the diffusion theory. In Section 3, it was revealed that we cannot essentially accept such diffusion concept also in view of the definition of diffusivity in a material. Nevertheless, the rela- 
tion of

$$
\tilde{D}=C^{\mathrm{I}} D_{\mathrm{INT}}^{\mathrm{II}}+C^{\mathrm{II}} D_{\mathrm{INT}}^{\mathrm{I}}
$$

proposed by Darken [35] in 1948 has been accepted as an actual one between the diffusivities of interdiffusion and intrinsic diffusion. The Darken Equation (55) has been widely used for analyzing interdiffusion problems since then [36]-[41]. However, it is self-evident that Equation (55) is entirely meaningless as mentioned above. Further, the Darken equation is also mathematically wrong from the beginning in the derivation process [42].

The Gauss theorem shows that Equation (20) gives the definition of diffusion flux and that the general formula of diffusion flux is expressed as Equation (21) in a fixed coordinate system. The Fick first law is thus incomplete one without an initial value. It is, therefore, obvious that the Fick first law is not worthy as a universal law now. Using not the Fick first law but the redefined diffusion flux of Equation (21), we can first reasonably understand the self-diffusion mechanism [22], regardless of the existing self-diffusion theory resulting from the tracer diffusion of isotope, where the diffusion of isotope used here should be essentially accepted as the impurity diffusion.

The problem of coordinate system for diffusion equation had never been discussed in the existing diffusion theory. The Gauss theorem reveals that the diffusion region space moves as shown in the relation between Equations (20) and (21). In other words, the discussion of coordinate transformation about the diffusion equations is indispensable for investigating the diffusion phenomena meaning the relative motion between micro particles. In relation to the matter mentioned here, the misconception of diffusion flux is discussed in the following.

It had been considered for a long time that the mathematical solutions of diffusion equation are impossible when the diffusivity depends on the concentration. Using the diffusion flux, therefore, the diffusion phenomena had been discussed until recently as there was no other choice except a numerical analysis. A diffusion flux similar to Equation (31) or (41) is proposed as

$$
J(t, x)=-D \partial_{x} C(t, x)+v_{\mathrm{F}} C(t, x),
$$

without a discussion about the coordinate system of diffusion equation [43] [44]. In relation to an external force in the diffusion field, Equation (56) was derived from investigating the difference of jumping frequencies between nearest-neighbor interfaces. In the derivation process, a velocity $v_{\mathrm{F}}$ called the drift velocity corresponds to the gradient of diffusivity. It has been also considered that the drift velocity depends on both the independent variables $t$ and $x$ [43]. However, as shown in equation (6), the gradient of diffusivity $\partial_{x} D$ not only has the dimension $\left[\mathrm{m} \cdot \mathrm{s}^{-1}\right]$ but also is a velocity itself in the local space concerned, and also Equations (33) and (39) give evidence that the $\partial_{x} D$ has the same dependence as the velocity between coordinate origins, which is dependent only on 
$t$. From the beginning, the diffusion flux should be essentially obtained by integrating both the sides of diffusion equation as defined by Equation (20). In that case, it is self-evident that there is no such a term relevant to the diffusivity gradient in the diffusion flux. As a result, we cannot physically accept such a drift velocity dependent on both $t$ and $x$. Hereafter, Equation (31) or (41) should take the place of Equation (56) in accordance with the coordinate transformation theory.

The matters relevant to the misconceptions pointed out here are extremely fundamental and they have been mistaken for such a long time in the history of diffusion. In fact, a lot of research papers based on the misconceptions are still published in the famous journals. Further, the misconceptions are also still plausibly described in many usual textbooks [43] [45] [46], just because of the fundamental matters for such a long term. They cause serious problems in the fields of diffusion research and education for younger people. Thus, the misconceptions should be made universally known for the development of diffusion research in future.

\section{Discussion and Conclusions}

The definition of diffusivity expressed by Equation (6) indicates that the quantum effect on the elementary process of diffusion should be essentially incorporated into the diffusivity as a characteristic of micro particle. The correlation between the Schrödinger equation and the diffusion equation revealed that the relation of material wave is reasonably valid regardless of the photon energy. Using the relation of material wave and the uncertain principle, we could reasonably understand the elementary process of diffusion through the different mechanism from the existing theory. At the same time, the universal diffusivity expression of Equation (14) was reasonably obtained using the elementary quantity $\left(5 \times 10^{2} N_{\mathrm{A}} \hbar=3.18 \times 10^{-8}\left[\mathrm{~m}^{2} \cdot \mathrm{s}^{-1}\right]\right)$ of diffusivity including the characteristic constant of a micro particle called the Planck constant $\hbar$ and that of a unit group of micro particles called the Avogadro constant $N_{\mathrm{A}}$.

The coordinate origin of basic diffusion equation expressing a relative motion between micro particles should be essentially set at a point of diffusion region space (or a virtual inert marker) on the initial interface shown in Figure 2. The raft model shows that the origin of coordinate $(\tilde{t}, \tilde{x})$ moves with a velocity expressed by Equation (46) as far as the initial condition of a given diffusion system satisfies the relation of

$$
\sum_{j=I}^{N} \sqrt{D_{\omega}^{j}}\left(C_{\mathrm{A}}^{j}-C_{\mathrm{B}}^{j}\right) \neq 0 .
$$

In that case, the basic diffusion equation is expressed by a moving coordinate system as discussed in Section 4. Here, we concluded again that the basic diffusion equation is

$$
\partial_{t} C=D\langle\tilde{\nabla} \mid \nabla C\rangle
$$


even if $\langle\tilde{\nabla}| D \neq 0$ is valid.

On the other hand, the diffusion equation of a fixed coordinate system is expressed as

$$
\partial_{t} C=\langle\tilde{\nabla} \mid D \nabla C\rangle .
$$

Here, it was concretely confirmed in Section 6 that solutions of Equations (7) and (18) are then transformable to each other by using Equation (44). In general, therefore, the basic equation of diffusion phenomena should be certainly defined as Equation (7).

Hereafter, Equation (7) will be widely applicable to the interdiffusion problems for the development of useful materials in future. In that case, however, we must then examine a phase shift resulting from the coordinate transformation of diffusion equation and further a phase shift caused by difference between a temperature during diffusion treatment and a room temperature after diffusion treatment.

In addition, as an especial case, if the relation of

$$
\sum_{j=I}^{N} \sqrt{D_{\omega}^{j}}\left(C_{\mathrm{A}}^{j}-C_{\mathrm{B}}^{j}\right)=0
$$

is valid, $f=0$ is also valid because of Equation (34). In that case, the Fick first law is then valid under the condition of $J_{\mathrm{eq}}=0$. At the same time, the Fick second law of Equation (18) is equivalent to Equation (7). In other words, the Fick laws are valid only under the extremely limited condition for diffusion problems.

In history, Fick applied the heat conduction equation to the diffusion phenomena as it had been. From a mathematical viewpoint on the Gauss theorem, however, we should have taken account of the difference between the state quantity of heat distribution and the real quantity of concentration profile of diffusion particles in those days. The mathematical misconceptions resulting from its thoughtlessness caused subsequently physical misconceptions on the fundamental theory in the diffusion problems as discussed in Section 7. They had been left untouched for such a long history of diffusion theory until recently.

In the present work, it was confirmed that the coordinate transformation of diffusion equation is indispensable for understanding the diffusion theory. The coordinate transformation theory shows that the general solutions (26) and (27) will be extremely useful for investigating the interdiffusion problems of many elements system in future.

The historical misconceptions such as an intrinsic diffusion and a drift velocity, which are actually nonexistent in the recent diffusion theory, were reasonably solved as concretely discussed in the present work. The new findings discussed here in accordance with the mathematical physics will be useful for understanding various diffusion problems in future, just because of the matters relevant to the fundamental theory. 


\section{References}

[1] Fick, A. (1855) Philosophical Magazine and Journal of Science, 10, 31-39. https://doi.org/10.1080/14786445508641925

[2] Fourier, J.B.J. (1822) Theorie Analytique de la chaleur. Didot, Paris, 499-508.

[3] Brown, R. (1828) Philosophical Magazine, 4, 161-173.

[4] Markov, A.A. (1960) American Mathematical Society Translations Series, 15, 1-14.

[5] Koch, A.J. and Meinhardt, H. (1994) Review Modern Physics, 66, 1481-1507. https://doi.org/10.1103/RevModPhys.66.1481

[6] Scholz, T. and Mandelknow, E. (2014) Cellular and Molecular Life Sciences, 71, 3139-3150. https://doi.org/10.1007/s00018-014-1610-7

[7] Serag, M. and Habuchi, S. (2017) Nature Communication, 8, Article No. 15675. https://doi.org/10.1038/ncomms15675

[8] Cottrell, G.W. (2016) Science, 313, 454-455. https://doi.org/10.1126/science.1129813

[9] Nakano, T., Okaie, Y. and Liu, J.Q. (2012) IEEE on Communication Letters, 16, 797-800. https://doi.org/10.1109/LCOMM.2012.042312.120359

[10] Taniguchi, M., Bando, M. and Nakayama, A. (2007) Journal of the Physical Society of Japan, 76, Article ID: 124003.

[11] Altman, E.I. and Schwarz, U.D. (2011) Advanced Materials, 22, 2854-2869. https://doi.org/10.1002/adma.200903927

[12] Novikov, D., Fieremans, E., Jensen, J.H. and Helpern, J.A. (2011) Nature Physics, 7, 508-514.

[13] Agarwal, K., Gopalakrishnan, S., Knap, M., Müller, M. and Demler, E. (2015) Physical Review Letters, 114, Article ID: 160401. https://doi.org/10.1103/PhysRevLett.114.160401

[14] Zaburdaev, V., Denisov, S. and Klafter, J. (2015) Review of Modern Physics, 87, 483. https://doi.org/10.1103/RevModPhys.87.483

[15] Lausch, T., Mayer, D., Schmidt, F., Lutz, E. and Widea, A. (2017) Nature Physics, 13, 137-141.

[16] Manukyan, L., Montandon, S.A., Fofonjka, A., Smirnov, S. and Milinkovitch, M.C. (2017) Nature, 544, 173-179. https://doi.org/10.1038/nature22031

[17] Schrödinger, E. (1926) Annals of Physics, 79, 361-376. https://doi.org/10.1002/andp.19263840404

[18] De Broglie, L. (1923) Nature, 112, 540. https://doi.org/10.1038/112540a0

[19] Okino, T. (2013) Journal of Modern Physics, 4, 612-615. https://doi.org/10.4236/jmp.2013.45088

[20] Boltzmann, L. (1872) Wiener Berichte, 66, 275-370.

[21] Okino, T. (2012) Journal of Modern Physics, 3, 1388-1393. https://doi.org/10.4236/jmp.2012.310175

[22] Okino, T. (2015) Journal of Modern Physics, 6, 2109-2144. https://doi.org/10.4236/jmp.2015.614217

[23] Smigelskas, A.D. and Kirkendall, E.O. (1947) Transactions of AIME, 171, 130-142

[24] Okino, T. (2014) Applied Physics Research, 6, 1-7. https://doi.org/10.5539/apr.v6n2p1

[25] Okino, T. (2011) Materials Transactions, 52, 2220-2227.

https://doi.org/10.2320/matertrans.M2011137 
[26] Cho, H., Yamada, K. and Okino, T. (2018) Journal of Modern Physics, 9, 130-144. https://doi.org/10.4236/jmp.2018.92009

[27] Dirac, P.A.M. (1958) The Principles of Quantum Mechanics. Oxford Clarendon Press, Oxford.

[28] Einstein, A. (1905) Annalen der Physik, 17, 549-560. https://doi.org/10.1002/andp.19053220806

[29] Heisenberg, W. (1927) Zeitschrift für Physik, 43, 172-198. https://doi.org/10.1007/BF01397280

[30] Boltzmann, L. (1894) Annual Review of Physical Chemistry, 53, 959-964. https://doi.org/10.1002/andp.18942891315

[31] Matano, C. (1933) Japanese Journal of Physics, 8, 109-113.

[32] Okino, T., Cho, H. and Yamada, K. (2017) Journal of Modern Physics, 8, 903-918. https://doi.org/10.4236/jmp.2017.86056

[33] Langevin, P. (1908) Comptes Rendus de Academie des Sciences (Paris), 146, 530-533.

[34] Okino, T. (2012) Journal of Modern Physics, 3, 255-259. https://doi.org/10.4236/jmp.2012.33034

[35] Darken, L.S. (1948) Transactions of AIME, 175, 184-201.

[36] Iijima, Y., Funayama, K., Kosugi, T. and Fukumichi, K. (1996) Philosophical Magazine Letters, 74, 423-428. https://doi.org/10.1080/095008396179959

[37] Masumura, R.A., Rath, B.B. and Pande, C.S. (2002) Acta Materialia, 50, 4535-4544. https://doi.org/10.1016/S1359-6454(02)00273-2

[38] Tsige, M.T. and Crest, G.S. (2004) Journal of Chemical Physics, 120, 2989-2995. https://doi.org/10.1063/1.1640347

[39] Horbach, J., Das, S.K., Griesche, M.P., Macht, M.P., Frohberg, G. and Meyer, A. (2007) Physical Review B, 75, Article ID: 174304. https://doi.org/10.1103/PhysRevB.75.174304

[40] Svoboda, J. and Fischer, F.D. (2011) Acta Materialia, 59, 1212-1219. https://doi.org/10.1016/j.actamat.2010.10.054

[41] Nathaniel, R., Scott, D.B. and Jerome, D. (2017) Physical Review E, 95, Article ID: 013206. https://doi.org/10.1103/PhysRevE.95.013206

[42] Okino, T. (2013) Journal of Modern Physics, 4, 1495-1498. https://doi.org/10.4236/jmp.2013.411180

[43] Manning, J.R. (1972) Theory of Diffusion, Diffusion 1-23. American Society for Metals, Metals Park.

[44] Yang, M. and Ripoll, M. (2012) Journal of Chemical Physics, 136, Article ID: 204508. https://doi.org/10.1063/1.4723685

[45] Shewmon, P.G. (1963) Diffusion in Solids. Series in Materials Science and Engineering, McGraw-Hill, New York.

[46] Mehrer, H. (2007) Diffusion in Solid. Springer-Verlag, Berlin Heidelberg. https://doi.org/10.1007/978-3-540-71488-0 